\title{
Optimal care for people affected by cancer
}

\section{Michael Jefford}

AUSTRALIA HAS THE HIGHEST per capita rate of cancer. In 2020, an estimated 145,000 Australians were diagnosed with cancer. ${ }^{1}$ An estimated 48,000 Australians died from cancer in the same year. However, five-year relative survival has improved from $51 \%$ for all cancers combined in $1987-91$ to $70 \%$ in $2013-17 .^{1}$ By international comparison, Australia has among the highest survival rates for many cancer types. ${ }^{2}$ Well over one million Australians have a personal history of cancer ('cancer survivors'). ${ }^{3}$ Over $10 \%$ of all Australians aged $>60$ years and over $23 \%$ of those aged $>80$ years are survivors.

Patients are at the centre of complex treatment pathways, which often involve a combination of surgery, radiation, chemotherapy, hormonal therapy, immunotherapy and targeted therapies. National Optimal Care Pathways describe recommended care for people with cancer from pre-diagnosis through to survivorship and end-of-life care (with specific guides for general practitioners [GPs]). ${ }^{4}$ However, care is often fragmented, and both patients and caregivers have many supportive care needs. ${ }^{5}$ Almost all patients have comorbid illness, which may be undertreated in the cancer setting.

This issue of Australian Journal of General Practice describes aspects of modern cancer care and underscores the importance of GPs, and others in the primary and community settings, to achieve optimal whole-person care. The Focus articles emphasise the experiences of people at risk of developing cancer, those living with and beyond cancer, and caregivers, highlighting the practical, emotional and psychological impacts.

Koczwara et al describe how GPs and other primary care providers can support personalised care that is integrated between providers and across different settings. ${ }^{6}$
GPs have an important role in the care of cancer survivors. ${ }^{5}$ Hart et al describe a number of common impacts from cancer and cancer treatments, specifically fear of cancer recurrence, financial toxicity and the impact on relationships, providing guidance on how to respond to these issues. ${ }^{7}$

Nowadays, over $80 \%$ of children survive cancer, although treatments are not benign, and survivors live with longstanding consequences. ${ }^{8}$ GPs have many roles including promoting positive health behaviours, supporting surveillance, and assessing and managing mental health.

A proportion of Australians carry increased risk of developing cancer. Forbes Shepherd et al report data from a series of young people with Li-Fraumeni syndrome living with, or at risk of developing, cancer, reflecting on potential lifelong cancer screening. ${ }^{9}$ They highlight the complex psychological situation for these young people and potential roles for the GP.

Family members, friends and colleagues are also impacted by cancer. Ugalde et al describe the challenges for caregivers. ${ }^{10}$ With a shift to more care being provided at home, and the greater complexity of care, caregivers take on a growing role in the management of people with cancer. GPs should acknowledge and support caregivers, identify caregivers' needs, and link them with supports.

Each Focus article underscores the essential role of general practice in the care of people at risk of and affected by cancer. Effort needs to be made to most effectively combine expert cancer care with holistic, whole-person care, across the cancer continuum. Clear communication is essential. Cancer specialists should provide guidance and clarify roles and responsibilities of all providers.

Non-government organisations, including cancer charities, patient support and community organisations, are important providers of information and support. More needs to be done to determine how these resources can be integrated most effectively to achieve best possible, equitable outcomes.

Cancer Australia is currently working to develop a patient-centred Australian Cancer Plan to meet the needs of Australians now and for the next 10 years. ${ }^{11}$ It is hoped this will further strengthen the vital role of primary care providers in contemporary cancer care.

\section{Author}

Michael Jefford MBBS, MPH, MHIthServMt, PhD, FRACP, Consultant Medical Oncologist, Department of Medical Oncology, Peter MacCallum Cancer Centre, Vic; Professor, Sir Peter MacCallum

Department of Oncology, University of Melbourne, Vic

\section{References}

1. Australian Institute of Health and Welfare. Cancer data in Australia. Cat. no: CAN 122. Canberra, ACT: AlHW, 2021.

2. Arnold M, Rutherford MJ, Bardot A, et al. Progress in cancer survival, mortality, and incidence in seven high-income countries 1995-2014 (ICBP SURVMARK-2): A population-based study. Lancet Oncol 2019;20(11):1493-505. doi: 10.1016/S14702045(19)30456-5

3. Cancer Council. Australians living with and beyond cancer in 2040. Sydney, NSW: Cancer Council Australia, 2018.

4. Cancer Council. Optimal cancer care pathways. Sydney, NSW: Cancer Council Australia, [date unknown].

5. Jefford M, Koczwara B, Emery J, Thornton-Benko E, Vardy JL. The important role of general practice in the care of cancer survivors. Aust J Gen Pract 2020;49(5):288-92. doi: 10.31128/AJGP-10-19-5133.

6. Koczwara B, Thornton-Benko E, Cohn RJ, et al. Personalised cancer care in the era of precision medicine. Aust J Gen Pract 2021;50(8):533-37.

7. Hart NH, Smith $A B$, Hobbs $K$, et al. Juggling cancer and life in survivorship: The role of general practitioners. Aust J Gen Pract 2021;50(8):520-25

8. McLoone J, Wakefield CE, Sansom-Daly UM, et al. The lived experience of children and adolescents with cancer. Aust J Gen Pract 2021;50(8):545-49.

9. Forbes Shepherd R, Keogh LA, Werner-Lin A, Delatycki MB, Forrest LE. Benefits and burdens of risk management for young people with inherited cancer: A focus on Li-Fraumeni syndrome. Aust J Gen Pract 2021;50(8):538-44

10. Ugalde A, Heynsbergh N, Sansom-Daly UM, et al. Effective integration of caregivers and families as part of the care team for people with cancer. Aust J Gen Pract 2021;50(8):527-31.

11. Cancer Australia. Australian Cancer Plan. Strawberry Hills, NSW: Cancer Australia, [date unknown]. 